\title{
Dynamic proteomic analysis of Aedes aegypti Aag-2 cells infected with Mayaro virus
}

\author{
Anna Fernanda Vasconcellos ${ }^{1,2+}$, Samuel Coelho Mandacaru ${ }^{1 \dagger}$, Athos Silva de Oliveira ${ }^{2}$, Wagner Fontes ${ }^{1}$, \\ Reynaldo Magalhães Melo ${ }^{1}$, Marcelo Valle de Sousa ${ }^{1}$, Renato Oliveira Resende ${ }^{2^{*}}$ and Sébastien Charneau ${ }^{1 *}$ (D)
}

\begin{abstract}
Background: Mayaro virus (MAYV) is responsible for a mosquito-borne tropical disease with clinical symptoms similar to dengue or chikungunya virus fevers. In addition to the recent territorial expansion of MAYV, this virus may be responsible for an increasing number of outbreaks. Currently, no vaccine is available. Aedes aegypti is promiscuous in its viral transmission and thus an interesting model to understand MAYV-vector interactions. While the life-cycle of MAYV is known, the mechanisms by which this arbovirus affects mosquito host cells are not clearly understood.

Methods: After defining the best conditions for cell culture harvesting using the highest virus titer, Ae. aegypti Aag-2 cells were infected with a Brazilian MAYV isolate at a MOI of 1 in order to perform a comparative proteomic analysis of MAYV-infected Aag-2 cells by using a label-free semi-quantitative bottom-up proteomic analysis. Time-course analyses were performed at 12 and $48 \mathrm{~h}$ post-infection (hpi). After spectrum alignment between the triplicates of each time point and changes of the relative abundance level calculation, the identified proteins were annotated and using Gene Ontology database and protein pathways were annotated using the Kyoto Encyclopedia of Genes and Genomes.
\end{abstract}

Results: After three reproducible biological replicates, the total proteome analysis allowed for the identification of 5330 peptides and the mapping of 459, 376 and 251 protein groups, at time 0, 12 hpi and 48 hpi, respectively. A total of 161 mosquito proteins were found to be differentially abundant during the time-course, mostly related to host cell processes, including redox metabolism, translation, energy metabolism, and host cell defense. MAYV infection also increased host protein expression implicated in viral replication.

Conclusions: To our knowledge, this first proteomic time-course analysis of MAYV-infected mosquito cells sheds light on the molecular basis of the viral infection process and host cell response during the first 48 hpi. Our data highlight several mosquito proteins modulated by the virus, revealing that MAYV manipulates mosquito cell metabolism for its propagation.

Keywords: MAYV, Mosquito, Infection, Virus-vector interaction, Semi-quantitative proteomics, Differentially expressed proteins

*Correspondence: rresende@unb.br; charneau@unb.br

${ }^{\dagger}$ Anna Fernanda Vasconcellos and Samuel Coelho Mandacaru contributed equally to this work

${ }^{1}$ Laboratory of Protein Chemistry and Biochemistry, Department of Cell Biology, Institute of Biology, University of Brasilia, Brasilia, DF 70910-900, Brazil

${ }^{2}$ Laboratory of Virology, Department of Cell Biology, Institute of Biology, University of Brasilia, Brasilia, DF 70910-900, Brazil

\section{Background}

Mayaro virus (MAYV) is an arbovirus (arthropodborne virus) that causes febrile illness and arthralgia in humans [1]. The virus belongs to the genus Alphavirus, family Togaviridae, sharing biological and medical aspects with other important arboviruses, such as chikungunya virus (CHIKV) [2]. MAYV is an emerging 
and not well recognized virus, so MAYV fever has been frequently misdiagnosed as other mosquito-borne diseases, including dengue virus (DENV) fever, due to their similar clinical symptoms [3]. The majority of countries that have reported autochthonous cases of MAYV are in Latin America [4].

MAYV has a positive single-stranded RNA genome of about $11.2 \mathrm{~kb}$ that encodes two polyproteins [5]. Both of them are cleaved by host and viral proteases in the cytoplasm, originating mature non-structural (nsP1, nsP2, nsP3 and nsP4) and structural (CP, E3, E2, $6 \mathrm{~K}$ and E1) proteins [6]. The non-structural proteins are directly translated from the genomic RNA and, in addition to other functions, catalyze viral RNA synthesis. The structural proteins are translated from a subgenomic RNA and assemble to form the virus particles [7].

Despite its restricted area of distribution, MAYV can be transmitted to humans by mosquitoes of at least four different genera, having a great potential for expansion. Haemagogus spp. are the main vectors, but transmission has also been reported from Culex spp., Sabethe spp. and Aedes spp. [4, 5]. Understanding the virus-vector interactions is one of the ways to develop strategies for virus control. Viruses are intracellular parasites with small genomes that hijack and manipulate the host cell machinery for their own replication $[8,9]$. In this context, host proteins perform important roles during the virus cycle and are key factors in understanding the steps involved in virus infection and therefore in developing methods in halting virus replication.

Aedes aegypti is well adapted to urban domestic habitats and has a strong human-feeding preference. Moreover, its widespread colonization and distribution in the tropics, has meant that this mosquito species has become highly adapted to urban tropical areas [10]. Aedes aegypti is very promiscuous concerning viral transmission, making it an interesting research model to understand virusvector interactions $[11,12]$. The availability of the $A e$. aegypti Aag-2 cell line also facilitates the establishment of infected cell cultures under controlled environmental conditions. In this study, we evaluated the proteome of Aag- 2 cells infected with MAYV by using label-free mass spectrometry. As a result, mosquito proteins that are important for MAYV replication have been identified, as well as proteins that may act as antiviral agents inhibiting virus replication.

\section{Methods}

\section{Cells and virus}

Vero cells (African green monkey kidney epithelial cells) were grown in high-glucose Dulbecco's modified Eagle's medium (DMEM; Sigma-Aldrich, St. Louis, Missouri,
USA) supplemented with $10 \%$ fetal bovine serum (FBS), and with $100 \mathrm{U} / \mathrm{ml}$ penicillin/streptomycin at $37{ }^{\circ} \mathrm{C}$ under $5 \% \mathrm{CO}_{2}$. Aedes albopictus $\mathrm{C6} / 36$ cells were grown in TC-100 medium (Vitrocell Embriolife, Campinas, SP, Brazil) supplemented with $10 \%$ FBS at $28{ }^{\circ} \mathrm{C}$. Aedes aegypti Aag-2 cells (provided by Gorben Pijlman from Wageningen University and Research, Wageningen, Netherlands) were grown in Schneider's medium (SigmaAldrich) supplemented with $10 \%$ FBS, and with $100 \mathrm{U} /$ $\mathrm{ml}$ penicillin/streptomycin at $28{ }^{\circ} \mathrm{C}$. Cell passages were performed every 3-4 days. The MAYV isolate (strain Campos-RJ, Brazil) was supplied by Instituto Oswaldo Cruz (Rio de Janeiro, Brazil). The MAYV isolate was first inoculated in a mammalian Vero cell monolayer culture, which is commonly susceptible and permissive to arboviruses and presents clear cytopathic effects (CPE) during the onset of virus replication.

\section{Mayaro virus propagation}

Once received, the MAYV isolate (in cell culture medium) was inoculated in Vero cells grown in a T25 flask. The supernatant was collected 3 days post-infection (dpi) following the onset of CPE. Virus replication was also confirmed by RT-PCR after RNA extraction of Vero cells with Trizol (Thermo Fisher Scientific, Waltham, MA, USA). For cDNA synthesis, SuperScript IV reverse transcriptase (Thermo Fisher Scientific) was used. PCR was then performed with Platinum Taq DNA polymerase (Thermo Fisher Scientific) with the degenerate primers (5'-GCR GCY YCG ACA GTG ACA GCY AT-3' and $5^{\prime}$-TGC ATG YGC TTT CGG TGC RC-3'), which amplified the E3-E2 genes (about $1.5 \mathrm{~kb}$ ). All procedures followed the manufacturers' recommendations. To increase the virus titer for further infection experiments using Aag-2 cells, Ae. albopictus C6/36 cells were infected with Vero-derived culture supernatant. These mosquito cells have a defective antiviral RNA interference response, which is advantageous for arbovirus propagation [13]. C6/36 supernatants were collected for virus titration and further use.

\section{Virus titration by end-point dilution assay}

Confluent Vero cells grown in T25 flasks were detached with $1 \mathrm{ml}$ trypsin-EDTA (Gibco, Carlsbad, CA, USA) and then diluted in $14 \mathrm{ml}$ supplemented DMEM. The Vero cells, in suspension, were incubated with serial dilutions (in supplemented DMEM) of virus stocks in a 1:1 ratio (90 $\mu \mathrm{l}$ cell suspension: $90 \mu \mathrm{l}$ virus dilution). A volume of $10 \mu \mathrm{l}$ per well was plated 6 -fold (per dilution) in 60 well Terasaki plates (Greiner Bio-One, Kremsmünster, Austria). Finally, the end-point dilution of infected cells was scored based on CPE visualized by light microscopy at 3-5 dpi. 


\section{Growth kinetics in Aag-2 cells}

To explore the proteomic changes of Aag-2 cells upon infection with MAYV, we first determined the best conditions for cell culture harvesting according to the highest virus titer. Aag-2 cells were infected in 6-well plates at MOI 0.1 and 1 in biological triplicates. After $90 \mathrm{~min}$ of adsorption, the supernatant was carefully removed and replaced with fresh Schneider's medium. The final MAYV-infected supernatant was titrated at 24, 48 and 72 hpi by end-point dilution assay (EPDA), and a virus growth curve was determined.

\section{Sample preparation for mass spectrometry}

Approximately $2 \times 10^{6}$ Aag- 2 cells were seeded in T25 flasks and infected with MAYV 16 h later at MOI 1, in triplicate. Cells, including mock-infected cells with Schneider's medium, were harvested at $12 \mathrm{hpi}$ and $48 \mathrm{hpi}$. Samples were reduced with $10 \mathrm{mM}$ dithiothreitol (DTT) in $0.25 \mathrm{M}$ Tris $\mathrm{pH} 8.6$ for $1 \mathrm{~h}$ at $52^{\circ} \mathrm{C}$ and then alkylated with $50 \mathrm{mM}$ iodoacetamide for $1 \mathrm{~h}$ at $25^{\circ} \mathrm{C}$. After digestion with trypsin (Promega, Madison, WI, USA) at a ratio 1:50 (enzyme:substrate), the samples were desalted through a pipette tip packed with a C18 membrane (PerSeptive Biosystems, Framingham, USA) and dried.

\section{Mass spectrometry}

An Orbitrap Elite ${ }^{\mathrm{TM}}$ hybrid ion trap-orbitrap mass spectrometer (Thermo Fisher Scientific) was used for LC-MS/ MS analysis. Samples were resuspended in $0.1 \%(\mathrm{v} / \mathrm{v})$ formic acid and loaded into a nano-UPLC-Dionex 3000 system (Thermo Fisher Scientific) equipped with a C18 trap type column $(100 \mu \mathrm{m} \times 3 \mathrm{~cm}$ with particles of $5 \mu \mathrm{m} / 100$ $\AA)$ and a C18 analytical column $(75 \mu \mathrm{m} \times 35 \mathrm{~cm}$ with particles of $3 \mu \mathrm{m} / 100 \AA$ ). Peptides were eluted from the analytical column with a gradient of $2 \%$ to $35 \%$ of solvent B (0.1\% (v/v) formic acid in acetonitrile) for $15 \mathrm{~min}$ and loaded directly into the mass spectrometer under electrospray ionization. Molecular mass spectra were acquired using the data-dependent acquisition (DDA) mode controlled by Xcalibur 2.0 software (Thermo Fisher Scientific). The DDA acquisition cycle comprised the range of $350-1650 \mathrm{~m} / \mathrm{z}$ and a resolution of 120,000 for MS1. The twenty most abundant precursor ions were fragmented by collision-induced dissociation (CID) during $90 \mathrm{~s}$ of dynamic exclusion and collision energy normalized at $35 \%$.

\section{Label-free protein quantification and identification}

For all biological replicates, quantification was performed using the Progenesis QI for proteomics software (version 1.0; Nonlinear Dynamics, Newcastle upon Tyne, UK). The spectra were aligned and quantified based on high mass accuracy MS1 events. Relative changes in abundance level were calculated for each detected MS1 event by comparing the peak areas calculated using extracted ion chromatogram. The changes were compared among LC-MS/MS runs according to the experimental design for replicates and conditions. No internal reference peaks were used. Fragment ion scans were exported as Mascot generic files (mgf) and searched with PEAKS Studio 7.0 (Bioinformatics Solutions Inc., Ontario, Canada) using the compiled UniProt "Ae. aegypti + MAYV" protein database $(25,023$ sequences accessed on 2 December 2018). The parameters used were: $10 \mathrm{ppm}$ peptide mass tolerance; $0.5 \mathrm{Da}$ fragment mass tolerance; and two missed cleavages allowed. Methionine oxidations and acetylation of protein $\mathrm{N}$-termini were specified as variable modifications, while carbamidomethylation of cysteine was specified as a fixed modification. Protein identities were assigned if at least two unique peptides were matched using a false discovery rate (FDR) of $<1 \%$. Identifications were re-imported into Progenesis QI, for protein quantification. Some identified peptides could be responsible for the identification of more than one protein, called conflicting peptides. These were detected and the most probable protein identification for each peptide was manually chosen according to the protein score, resolving the conflicts. Peptides still in conflict after manual validation were excluded from protein quantification.

\section{Statistical analysis and biological context}

All statistical analysis was performed on protein relative abundance data obtained through Progenesis QI software (Nonlinear Dynamics; http://www.nonlinear.com/ progenesis/qi). After normalization and transformation, data were analyzed by principal components analysis ( $\mathrm{PCA}$ ) provided in the $\mathrm{R}$ programming environment [14], through the FactoMineR package [15]. PCA was performed at both peptide and protein levels to evaluate whether the grouping among replicates was more evident than the grouping of conditions, as well as to detect for the possible presence of outliers. Three technical replicates were carried out for each sample preparation to confirm reproducibility. Proteins were considered significantly regulated among conditions based on ANOVA with $P$-values $P<0.05$. After being identified, the protein sequences were submitted to Gene Ontology annotation by Blast2GO software (BioBam Bioinformatics, Valencia, Spain) and to BlastKOALA in the Kyoto Encyclopedia of Genes and Genomes (KEGG- http://www.kegg. jp/blastkoala/) for functional annotation. Metaboanalyst 4.0 (https://www.metaboanalyst.ca/) was used to normalize and cluster the data, as well as to perform PCA analysis and plot heatmap graphs [16]. To better understand protein dynamics, the samples from the heatmap 
were clustered using the VSClust online platform (http:// computproteomics.bmb.sdu.dk/Apps/VSClust/), a clustering tool based on fuzzy c-means clustering that considers the variance of each feature [17].

\section{Results and discussion}

Despite Mayaro fever outbreaks having been recorded in Brazil, Bolivia and Peru since 1978 [18] and despite the recent emergence of MAYV recombinants that are more adapted to humans in Brazil and Haiti [19], the MAYV infection ability and its molecular pathology remain obscure. A label-free semi-quantitative bottom-up proteomic analysis over-time was performed to see how $A e$. aegypti Aag-2 cells respond to MAYV infection during different time points of infection.

\section{Infection of Aedes aegypti Aag-2 cells with MAYV}

The highest MAYV titer obtained when infecting Aag-2 cell culture was about $2 \times 10^{3} \mathrm{TCID}_{50} / \mathrm{ml}$ at MOI 1 and $48 \mathrm{hpi}$ (Fig. 1). At this time point (48 hpi), a two-fold change of infectious viruses was observed between MOI 0.1 and 1 . Based on these observations, the infection for proteomic analysis was carried out at MOI 1 and cell harvesting was performed at $12 \mathrm{hpi}$ and $48 \mathrm{hpi}$.

\section{Dataset analysis}

Following mass spectrometry, the PCA revealed that the data clustered more tightly with their replicates rather than experimental conditions (Fig. 2). The variable correlation plot indicates a large number of variables of good quality contributing to the formation of the principal components (Additional file 1: Figure S1).

The abundance variation pattern of all identified proteins over the different infection time points was visualized in a heatmap (Fig. 3a) from which four clusters emerged (Fig. 3b and Additional file 2: Table S1). The optimal number of clusters was defined according to the

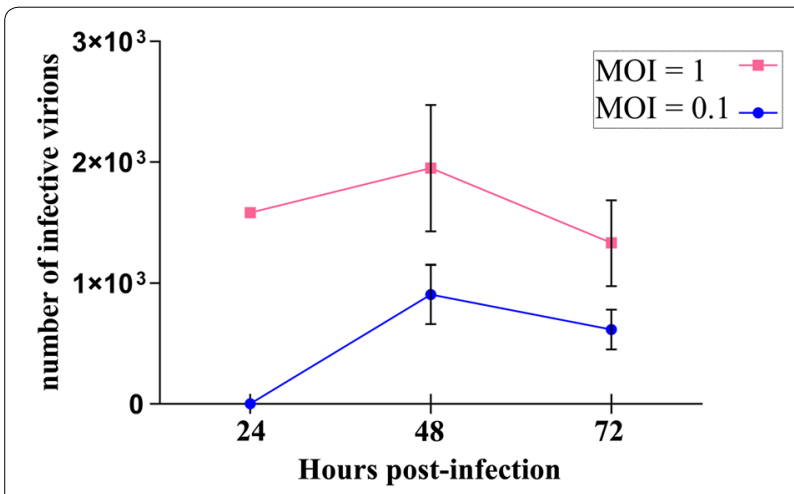

Fig. 1 MAYV growth kinetics in Aag-2 cells. Aag-2 cells were infected with MAYV at a MOI of 1 and were harvested at the indicated time points. Graphics obtained using GraphPad Prism 6 minimum centroid distance of each number of clusters tested (Additional file 3: Figure S2).

The clustering patterns highlighted protein similarity across the groups. Proteins in cluster 1 demonstrated similar abundances in control and $12 \mathrm{hpi}$, but increased in $48 \mathrm{hpi}$. A total of 33 proteins were grouped in this cluster. Protein abundance in cluster 2 decreased compared to control at $12 \mathrm{hpi}$ and $48 \mathrm{hpi}$. This cluster represented 20 proteins. In cluster 3 , protein abundance increased at $12 \mathrm{hpi}$ compared to the control and drastically decreased from $12 \mathrm{hpi}$ to $48 \mathrm{hpi}$, grouping 17 proteins. Cluster 4 demonstrated progressively increased abundance from control to $12 \mathrm{hpi}$, then to $48 \mathrm{hpi}$ with a slight decrease in slope, representing 11 proteins (Fig. 3b, Additional file 1: Figure S1).

\section{Global proteomics of MAYV-infected Aag-2 cells}

From three time-course infection experiments, three biological samples were harvested at each time point $(0$, $12 \mathrm{hpi}$ and $48 \mathrm{hpi}$ ). The total proteome analysis allowed the identification of 5330 peptides (Additional file 4: Table S2) providing a total of 564 non-redundant proteins of Aag- 2 cells. The analysis yielded the identification of 459, 378 and 253 protein groups, at time 0 (corresponding to uninfected Aag-2 cells, as a control), $12 \mathrm{hpi}$ and 48 hpi, respectively. A full list of the identified proteins is given in Additional file 5: Table S3. While the number of identified Ae. aegypti proteins drastically decreased, the number of identified MAYV proteins increased along the $48 \mathrm{~h}$ of infection (Fig. 4). During the viral infectious cycle, changes in represented insect cell pathways reflected the onset of the fast viral replication that explain an increase of viral protein abundance. Moreover, part of the infected cells in culture died, and consequently their proteins were progressively released in the culture medium from disrupted cells or degraded by proteolysis, explaining the decrease of the identification amount at the post-infection time points.

\section{Dynamic proteomics of MAYV-infected Aag- 2 cells}

A relative analysis of the changes in mosquito protein abundance between the three time points was performed using proteins that could be identified based on having two matching peptides. The abundance of 161 proteins changed significantly over the time course of infection (ANOVA $P$-values $<0.05$ ) (Additional file 6: Table S4). Thus, the changes observed in the Aag-2 cell proteome were likely to be related to MAYV infection effects. Many of the protein changes we observed have been recorded from previous infection experiments with arboviruses $[20,21]$. These include heat-shock proteins, ATP synthase and enolase phosphatase e1 (see below). 


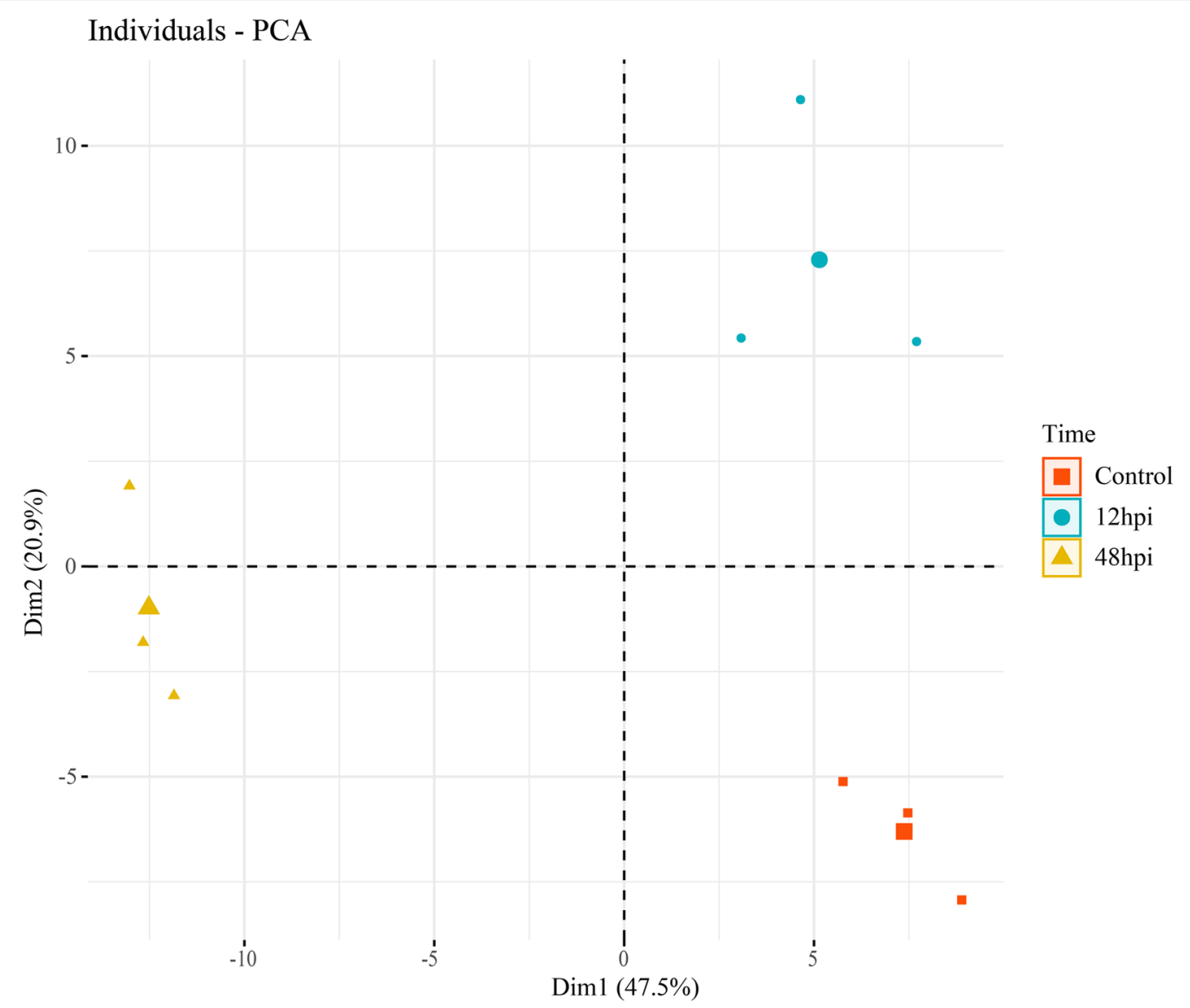

Fig. 2 Principal components analysis of the proteomic dataset. Orange squares are triplicate measurements at time 0 (corresponding to uninfected Aag-2 as control), blue dots and yellow triangles are the triplicates at times $12 \mathrm{hpi}$ and $48 \mathrm{hpi}$, respectively. The larger geometric symbols represent the centroid of each cluster triplicates. Graphics obtained using factorextra R Package, R environment

The Gene Ontology (GO) classifications of the modulated proteins according to cellular distribution and biological process are shown in Fig. 5a, b and listed in Additional file 6: Table S4. A large number of modulated proteins were related to bio-membranes, showing that our sample preparation method was efficient in recovering hydrophobic proteins. Proteins belonging to integral membrane component, organelle membrane and membrane complex categories comprised $31 \%, 5 \%$ and $6 \%$ of the 289 proteins identified, respectively. Other proteins were mainly assigned to cytosol or mitochondrion categories (17\% each) (Fig. 5a). The modulated proteins were mostly related to redox processes (29\%), translation (27\%) and other metabolic processes of compounds. The metabolism of phosphate-containing compounds represented 13\% of these (Fig. 5b). Therefore, the effect of the infection on the mosquito proteome was mostly associated with response to oxidative stress, homeostasis and protein folding, protein synthesis and ATP production.
Interestingly, Ae. aegypti proteins grouped in clusters 1, 3 and 4 (Fig. 3b) showed increased abundance at $12 \mathrm{hpi}$ or $48 \mathrm{hpi}$ against the overall trend of decreasing representation of mosquito proteins as the infection progressed.

Globally, Aag-2 cell proteome change was more clearly identified at 48 hpi than 12 hpi compared to time 0 . Given that the time required for replication of a single alphavirus genome is $4 \mathrm{~h}$ [20], the mosquito protein abundance variations may be associated with the increasing burden of infection.

\section{Viral particle internalization}

It is well known that arboviruses such as MAYV can infect both mosquito and vertebrate cells, suggesting that the host cell receptors involved with its internalization are shared between these cell types [21]. Besides virus internalization into insect and mammalian cells is mostly dependent on clathrin-mediated endocytosis, other surface receptors have been implicated in viral cell entrance [20]. In this study, prohibitin was upregulated 


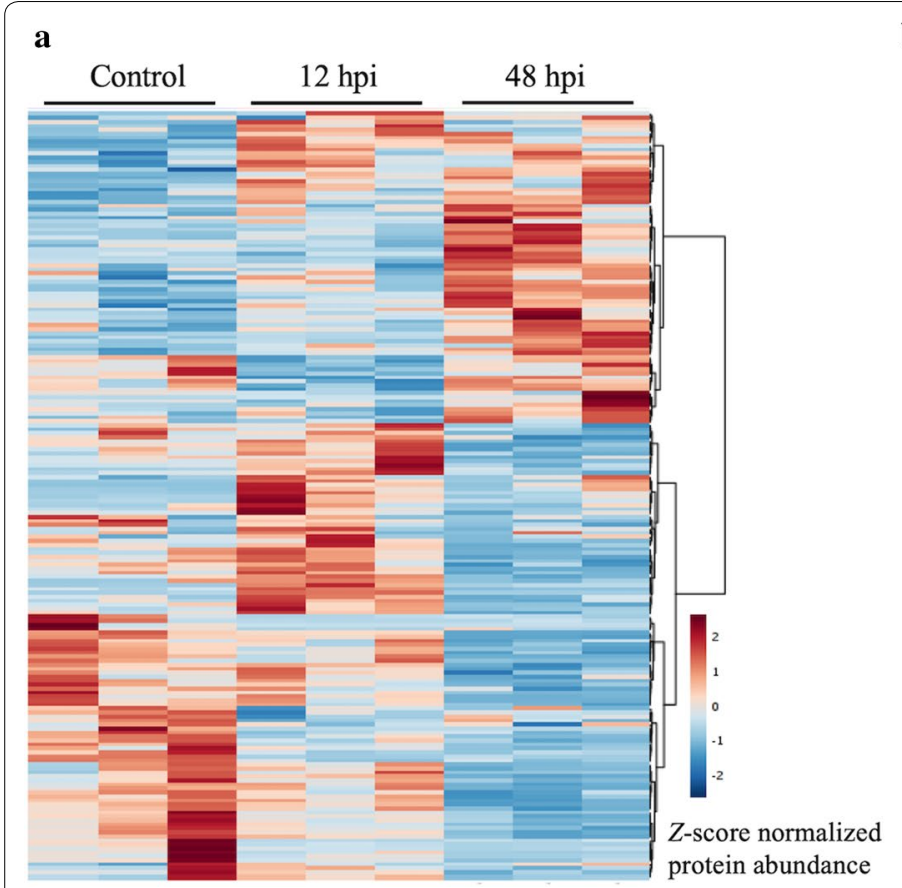

b
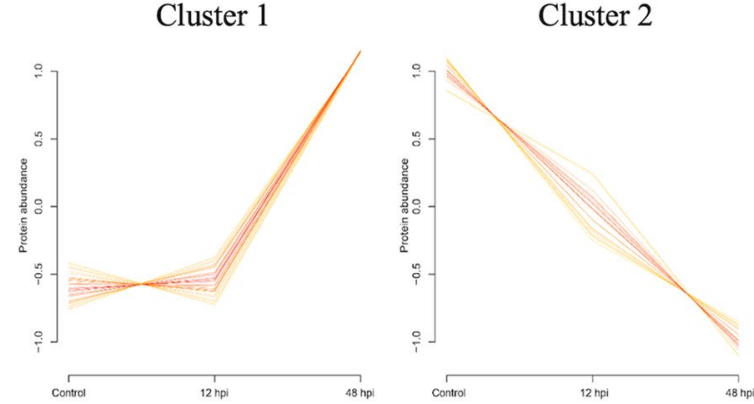

Cluster 3
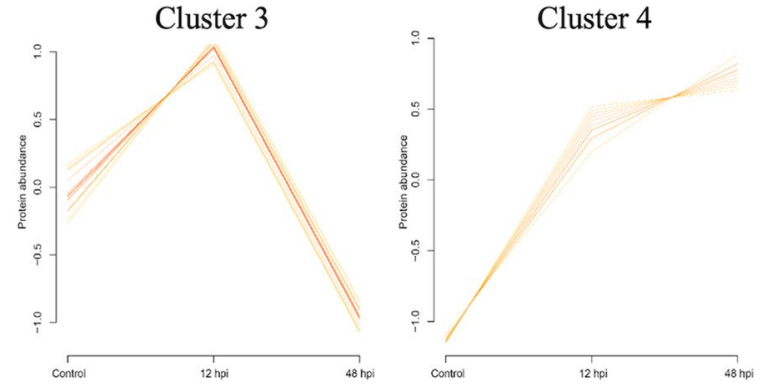

Fig. 3 Abundance of all Aag-2 quantified proteins over the different infection time points. a Heatmap presenting all Aag-2 quantified proteins. Graphics obtained using MetaboAnalyt 4.0. Proteins hierarchically clustered across all samples as shown on the right. b Protein dynamic abundance clusters over condition groups obtained through the VSClust algorithm, where the protein abundance is represented by a log-transformed normalized value

in Ae. aegypti cells at 48 hpi (Additional file 6: Table S4). This surface receptor has already been characterized as a mediator of dengue virus serotype 2 entrance into $A e$. albopictus and Ae. aegypti cells [22]. Prohibitin is also a mediator entrance of alphaviral CHIKV particles into mammalian cells from different lineages, even though its precise role is still unknown [23]. Thus, we propose that prohibitin may also mediate MAYV virus internalization in mosquito cells. However, given its ability to infect different cells, we cannot discard the possibility that MAYV may have other receptors and internalization mechanisms.

\section{Cell response to stress}

The heat-shock proteins (HSPs) are a large family of widely abundant and evolutionarily conserved molecular chaperones that act in cellular homeostasis, mainly in response to stress [24]. They act in the correct folding, assembly and traffic of proteins and protein complexes. Upregulation of chaperone expression, in general, can be triggered by different stimuli such as elevated temperatures, nutrient shortages or viral infections [25]. Here, HSP-20 and HSP-60 of Aag-2 cells showed increasing abundance throughout the infection (Additional file 6: Table S4). It was previously shown that, by increasing the incubation temperature of MAYV-infected Ae. albopictus C6/36 cells, the expression of HSPs was induced and viral replication was strongly inhibited [26]. More recently, C6/36 cells infected with the alphavirus CHIKV also induced overexpression of HSP-60 [27]. It was hypothesized that these chaperones could be acting in favor of viral replication. However, after knockout of this siRNA-mediated chaperone, C6/36 cells showed an increase in viral titre [27]. Moreover, transcriptomic analysis of Drosophila cell culture showed the induction of chaperones when infected with RNA viruses [28]. Altogether, these data point to a conserved antiviral mechanism and corroborate that chaperones are important in the response against MAYV in Ae. aegypti cells. Beyond this, one HSC70, the heat-shock protein 70 cognate 5, was also identified as upregulated in Aag-2 cells at 12 hpi with MAYV (Additional file 6: Table S4). The HSC70/HSP90 machinery is an important mediator of the RNA-induced silencing complex (RISC) assembly pathway for the RNA interference (RNAi) antiviral defense mechanism, helping the conformational opening of Argonaute proteins to receive small RNA duplexes in an ATP dependent manner [29]. Since the RNAi machinery is the main antiviral mechanism in invertebrates, the super expression of HSC70 may be a defense mechanism of the infected Aag- 2 cells. In fact, 


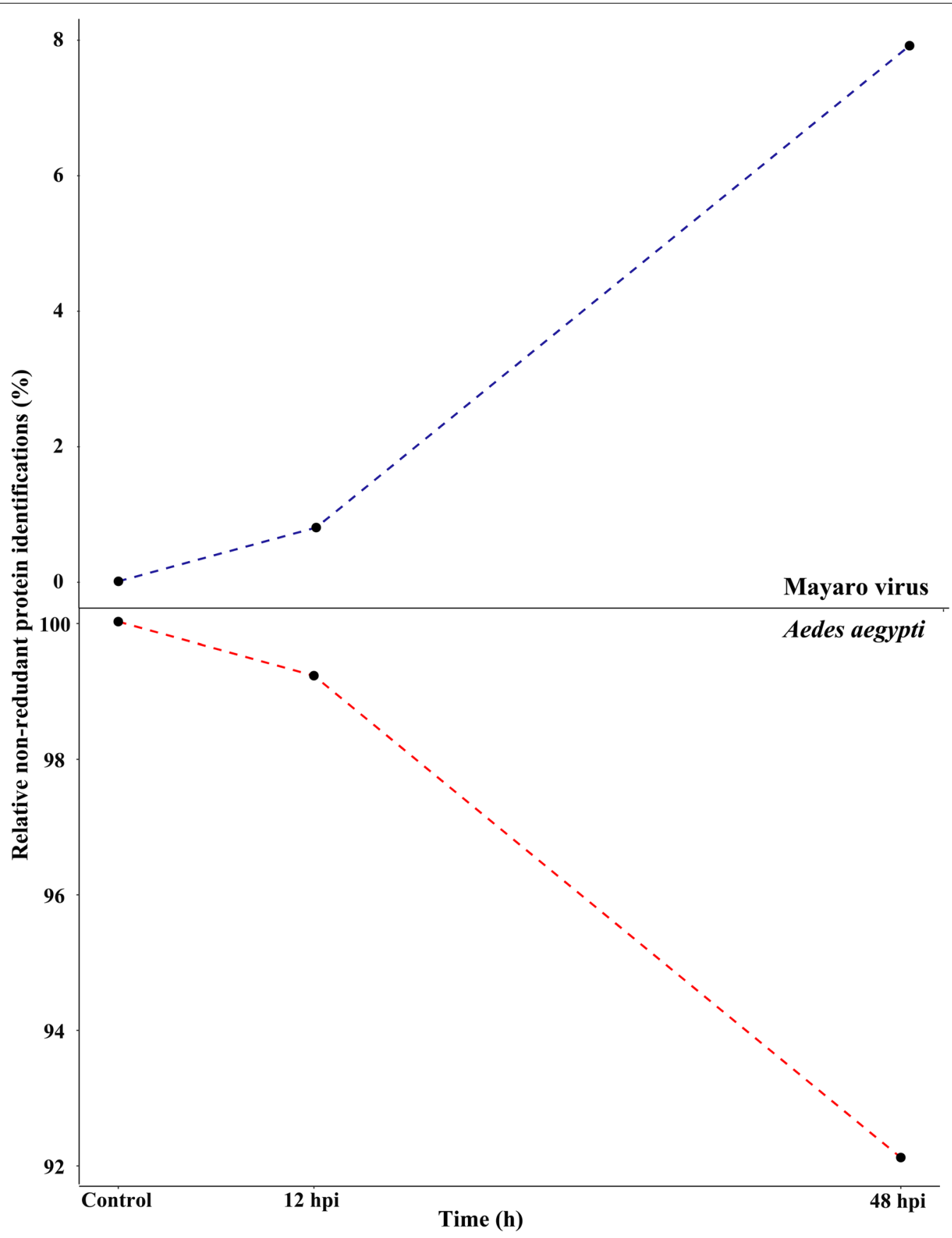

Fig. 4 Relative non-redundant protein identifications (\%) from MAYV (blue) and Aedes aegypti Aag-2 cells (red) over the different infection time points. Graphics obtained using R environment

Anopheles gambiae HSC70B has been implicated in viral replication blocking when infected with the togavirus O’Nyong-Nyong [30].

- Another protein associated with stress response is enolase phosphatase e1 (ENOPH1). Positively regulated throughout MAYV infection (Additional file 6: Table S4), ENOPH1 is an enzyme involved in L-methionine biosynthesis via the salvage pathway. This process catalyzes the conversion of methyladenosine back to L-methionine [31], and results in the production of polyamines, small cationic molecules essential for cell development and homeostasis [32]. The overexpression of ENOPH1 has already 

membrane protein complex
mitochondrion
nuclear part
organelle membrane
polymeric cytoskeletal fiber
integral component of membrane
cytosol
ribosomal subunit

\section{Cellular component}
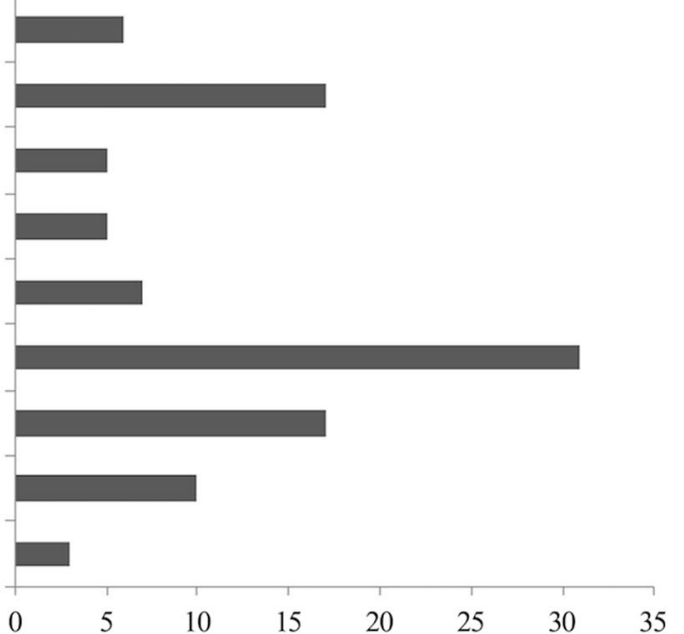

$\mathbf{b}$

\section{Biological process}

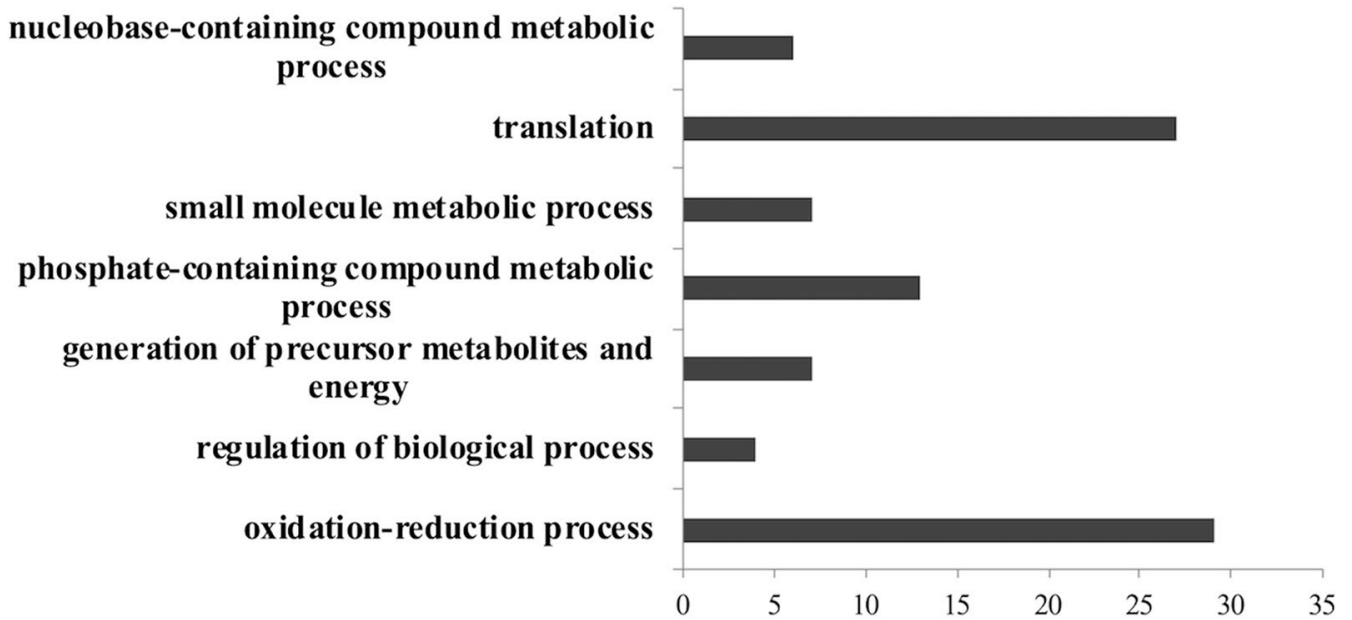

Fig. 5 Functional annotation of 161 proteins significantly differentially expressed in response to MAYV infection of Aag-2 cells. Bar charts represent the percentages of proteins associated to GO terms cellular component (a) and biological process (b), obtained using the software Blast2Go

been found in Ae. albopictus cell culture infected with DENV and with CHIKV [27, 33]. However, the CHIKV titer increased in knockout mosquito cell lines of the ENOPH1 gene, implying a function in regulating the stress response and innate immunity of host cells during infection. [27]. Therefore, the importance of the host ENOPH1 factor for viral replication could indicate a higher production of polyamines during infection.

\section{Energy and intermediate metabolite demand}

Both glucose and glutamine have been characterized as carbon sources for human viruses [34]. In this study, nine modulated Ae. aegypti enzymes that act in glycolysis were identified (Fig. 6, Additional file 6: Table S4). According to their pattern of protein abundance at 48 hpi with MAYV, increased glycolytic activity was implied, as well as an accumulation of metabolic intermediates. The decreased abundance of fructose 1,6-biphosphatase, phosphoglycerate kinase and phosphoglycerate mutase associated with an increased abundance of fructosebiphosphate aldolase and triosephosphate isomerase could have favored glyceraldehyde-3-phosphate accumulation. This glycolytic intermediate can be easily isomerized to dihydroxyacetone phosphate (DHAP), which is also involved in lipid production. Moreover, the pentose phosphate pathway, required for the synthesis of 


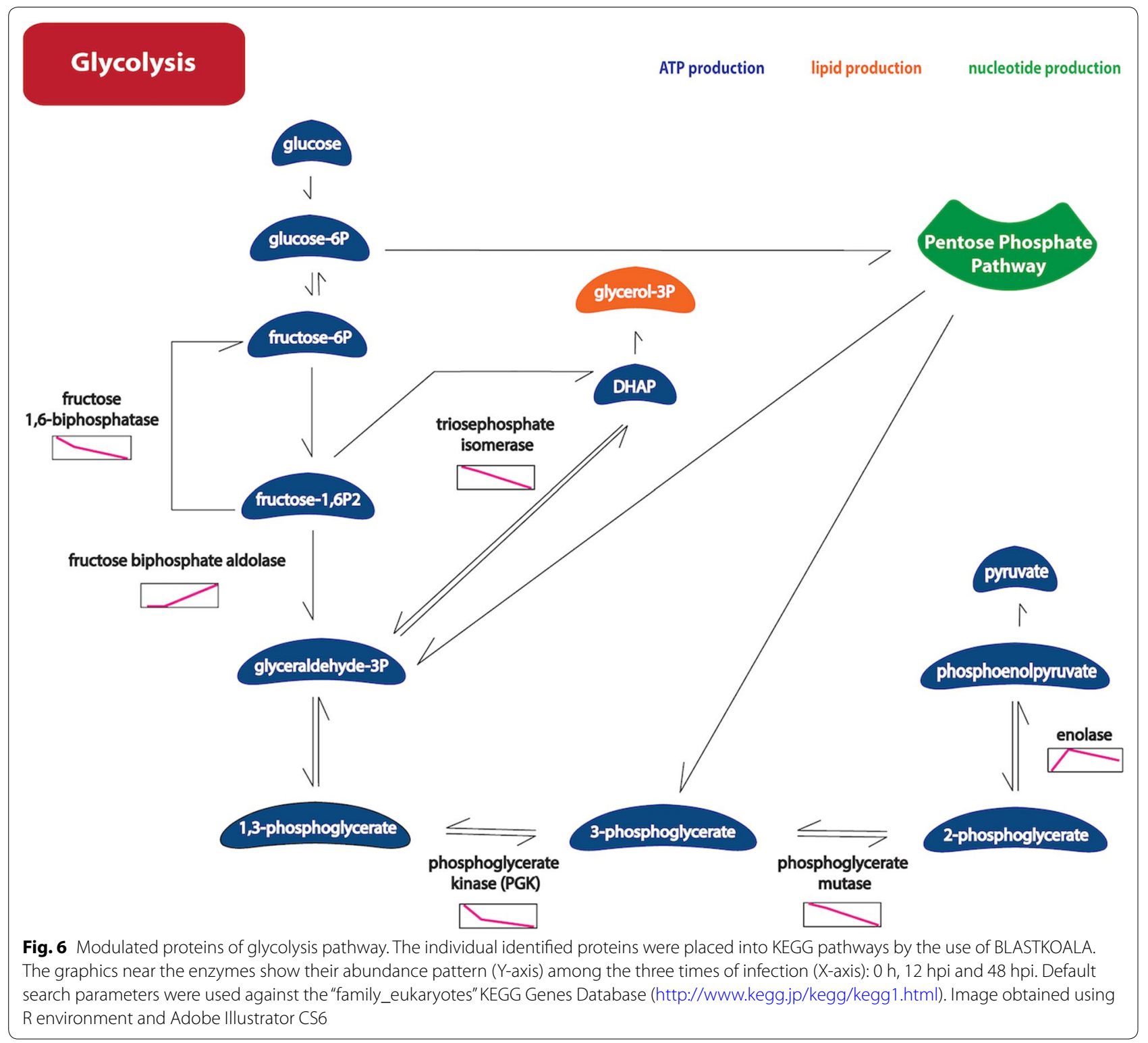

ribonucleotides and the production of the reducing agent NADPH, branches glyceraldehyde-3-phosphate from glycolysis. However, the increased abundance of the key glycolytic enzyme enolase may also be associated with the increase of glycolytic activity. The alterations in the proteins involved in the glycolytic pathway are probably not only due to rapid ATP production due to the high energy demand of the MAYV infectious cycle. Alphaviruses replicate their genome in association with cellular membranes and the budding of new virions can also occur in internal vesicles [35]. Therefore, nucleotide and lipid production could also have been activated by MAYV to facilitate its replication in Aag-2 cells. Furthermore, mitochondrial membrane disruption, with a consequent decrease in ATP levels, has been observed in influenza virus-infected cells, followed by glycolysis activation to reset energy levels [36]. For MAYV, another evidence of this energy demand is the overexpression of the Ae. aegypti alpha subunit of ATP synthase at 48 hpi (Additional file 6: Table S4). In support of this observation, $A e$. albopictus cell culture infected with CHIKV showed an increase in the beta subunit of ATP synthase expression [27].

Replication complexes and the budding of new virions Vesicle-associated membrane proteins (VAMPs) and an Aag-2 protein from the synaptobrevin family are among 
the most abundant proteins in 48 hpi (Additional file 6: Table S4). VAMPs are integral membrane components that mediate vesicle secretion [37]. In the process of new particle maturation, alphaviruses are assembled in vacuoles of the exocytic pathway in mosquito cells [38]. Jose et al. [35] have shown that alphavirus budding occurs on both the plasma membrane and internal vesicles. Thus, the budding of new viral particles by exocytic pathways may explain an increasing expression of VAMPs at $12 \mathrm{hpi}$ and at $48 \mathrm{hpi}$. According to the growth kinetics of MAYV in Aag-2 cells, the highest production of infectious viral particles was observed at $48 \mathrm{hpi}$, corresponding to the peak abundance of VAMP.

\section{Nuclear transport}

At 12 hpi, the host cell transcription factor C1 (HCFC1) was upregulated in Ae. aegypti cells. (Additional file 6: Table S4) In humans, HCFC1 is involved in the transcription of immediate herpes simplex virus genes [39]. In this context, human HCFC1 forms a protein complex with the viral DNA still in the cytoplasm mediated by the viral protein 16 (VP16) and directs it to the nucleus, commanding the expression of the immediate proteins [39]. According to Freiman and Herr [40], the association VP16-HCFC1 also occurs in invertebrates, suggesting that it is an evolutionarily conserved interaction. For example, even though herpes simplex virus has a DNA genome and nuclear replication, it was detected that RNA viruses with cytoplasmic replication infecting Drosophila also have a nuclear step [41]. Viral RNA was reverse-transcribed by the host cell and incorporated into genomic DNA as retrotransposon sequences to activate the RNAi pathway [41]. Thus, even in RNA viruses with cytoplasmic replication, it is possible that a nuclear step can be performed by the host cell as an antiviral mechanism. Although, as far as we know, no study has focused on this process involving HCFC1 during MAYV infection, this hypothesis should be investigated, given RNAi is the main antiviral mechanism in invertebrate cells.

\section{Conclusions}

To better understand the mechanisms by which the Mayaro virus affects mosquito host cells, we performed a comparative proteomic analysis of MAYV-infected Aag-2 cells during the time-course at $12 \mathrm{hpi}$ and $48 \mathrm{hpi}$. By mass spectrometry analysis, we identified 161 mosquito regulated proteins during MAYV infection. This proteome analysis of Ae. aegypti Aag-2 cells showed that differentially regulated proteins could be grouped into four clusters based on patterns of abundance change during the time course of infection. The results strongly suggest that the mosquito regulated proteins may be associated with the major infective events of MAYV in the host cell, from virus internalization, cell response to stress, energy demand, and new particle maturation. Some of our findings showed an increased expression of proteins such as prohibitin, synaptobrevin, transcription factor HCFC1, HSPs and enolase phosphatase e1, suggesting an important role of these proteins during MAYV infection. We also show that the glycolysis pathway was strongly modulated upon infection of insect cells indicating that infection is associated with a substantial alteration of host metabolism. Overall, this study provides a comprehensive understanding of the molecular basis of MAYV infection in Ae. aegypti Aag-2 cells by identifying several potential proteins and pathways directly related to the infection and consequent regulation of biochemical processes.

\section{Supplementary information}

Supplementary information accompanies this paper at https://doi. org/10.1186/s13071-020-04167-2.

Additional file 1: Figure S1. Variable correlation plot for PCA demonstrating the quality of each variable represented by the distance of the arrow and the origin. The color intensity indicates the contribution of a variable to the components formation and the distance of each arrow from the origin indicates the quality of this feature.

Additional file 2: Table S1. Protein accession numbers of the 4 clusters identified through the VSClust algorithm corresponding to Fig. 3a.

Additional file 3: Figure S2. Estimation of optimal number of clusters according to the protein abundances over time. The optimal number is defined by the minimum centroid distance, indicated by the black square on the image. The optimal number was used to perform the clustering in Fig. 3a. Analysis performed in the VSClust online platform.

Additional file 4: Table S2. All peptides from the three different infection time points ( 0 as control, $12 \mathrm{hpi}$ and $48 \mathrm{hpi}$ ), providing protein identification. Parameters used: FDR criterion of $1 \%$ and at least two unique peptides.

Additional file 5: Table S3. Aedes aegypti Aag-2 cell and Mayaro virus proteins identified over the different infection time points.

Additional file 6: Table S4. Aedes aegypti Aag-2 cell proteins with modulated abundance over the different infection time points and classification by $\mathrm{GO}$ terms for cellular component and biological process, obtained using the software Blast2Go corresponding to Fig. 5.

\section{Abbreviations}

ATP: Adenosine triphosphate; cDNA: Complementary DNA; CHIKV: Chikungunya virus; CPE: Cytopathic effects; DDA: Data-dependent acquisition;

DENV: Dengue virus; DMEM: Dulbecco's modified Eagle medium; Dpi: Day(s) post-infection; DTT: Dithiothreitol; EDTA: Ethylenediamine tetraacetic acid: ENOPH1: Enolase phosphatase e1; EPDA: End-point dilution assay; FBS: Fetal bovine serum; FDR: False discovery rate; GO: Gene Ontology; HCFC1: Host cell transcription factor C1; Hpi: Hour(s) post-infection; HSC: Heat shock cognate; HSP: Heat-shock protein; KEGG: Kyoto Encyclopedia of Genes and Genomes; LC-MS/MS: Liquid chromatography tandem mass spectrometry; MAYV: Mayaro virus; MOI: Multiplicity of infection; PCA: Principal components analysis; RISC: RNA-induced silencing complex; RNA: Ribonucleic acid; RNAi: RNA interference; RT-PCR: Reverse transcription polymerase chain reaction; VAMPs: Vesicleassociated membrane proteins; VP16: Viral protein 16.

Acknowledgements

We thank Nuno Domingues and Jaques M. F. Souza for technical assistance. 


\section{Authors' contributions}

AFV and SCM designed the study, performed the experiments, analyzed the proteomics data and drafted the manuscript. ASO designed the study and critically revised the manuscript. WF and RMM analyzed the proteomics data and revised the manuscript. ROR and MVS provided equipment and revised the manuscript. ROR and SC designed the study. SC also analyzed the proteomics data, drafted and critically revised the manuscript. All authors read and approved the final manuscript.

\section{Funding}

This work was supported by FAPDF (Demanda Induzida-Aedes aegypti e as arboviroses Zika, chikungunya e dengue 0193.000417/2016 to ROR; Demanda Espontânea 193.001.723/2017 to SC; fellowship to SCM), CNPq (Universal 430610/2016-5 to SC; fellowship to AFV), CAPES (COFECUB Grant 923/18 to SC), CAPES/ProEx, FINEP (CT-Infra Grants 0439/11 and 0694/13 to MVS)

\section{Availability of data and materials}

Data supporting the conclusions of this article are included within the article and its additional files. Mass spectrometer output files (raw data) are available from the MassIVE database (accession number MSV000084687, https ://doi.org/10.25345/c5h67w, https://massive.ucsd.edu/ProteoSAFe/datas et.jsp?task=da6985a8dcdd47b0aa0a8bc105c814c0) and ProteomeXchange (accession number PXD016737) [42-44].

\section{Ethics approval and consent to participate}

Not applicable.

\section{Consent for publication}

Not applicable.

\section{Competing interests}

The authors declare that they have no competing interests.

Received: 10 January 2020 Accepted: 2 June 2020

Published online: 10 June 2020

\section{References}

1. Cavalheiro MG, Costa LS, Campos HS, Alves LS, Assuncao-Miranda I, Poian AT. Macrophages as target cells for Mayaro virus infection: involvement of reactive oxygen species in the inflammatory response during virus replication. Ann Acad Bras Ciênc. 2016;88:1485-99.

2. Figueiredo ML, Figueiredo LT. Emerging alphaviruses in the Americas: chikungunya and Mayaro. Rev Soc Bras Med Trop. 2014;47:677-83.

3. Mota MT, Terzian AC, Silva ML, Estofolete C, Nogueira ML. Braz. J Microbiol. 2016;47(Suppl. 1):38S-50S.

4. Acosta-Ampudia Y, Monsalve DM, Rodriguez Y, Pacheco Y, Anaya JM, Ramirez-Santana C. Mayaro: an emerging viral threat? Emerg Microbes Infect. 2018;7:163.

5. Napoleão-Pego P, Gomes LP, Provance DW Jr, De Simone SG. Mayaro virus disease. J Hum Virol Retrovirol. 2014;1:00018.

6. Sudeep AB, Parashar D. Chikungunya: an overview. J Biosci. 2008:33:443-9.

7. Solignat M, Gay B, Higgs S, Briant L, Devaux C. Replication cycle of chikungunya: a re-emerging arbovirus. Virology. 2009;393:183-97.

8. Sanchez EL, Lagunoff M. Viral activation of cellular metabolism. Virology. 2015:479:609-18

9. Gonzalez Plaza JJ, Hulak N, Kausova G, Zhumadilov Z, Akilzhanova A. Role of metabolism during viral infections, and crosstalk with the innate immune system. Intractable Rare Dis Res. 2016;5:90-6.

10. McBride CS, Baier F, Omondi AB, Spitzer SA, Lutomiah J, Sang R, et al. Evolution of mosquito preference for humans linked to an odorant receptor. Nature. 2014:515:222-7.

11. Powell JR, Tabachnick WJ. History of domestication and spread of Aedes aegypti - a review. Mem Inst Oswaldo Cruz. 2013;108(1):11S-7S.

12. Franz AW, Kantor AM, Passarelli AL, Clem RJ. Tissue barriers to arbovirus infection in mosquitoes. Viruses. 2015:7:3741-67.

13. Brackney DE, Scott JC, Sagawa F, Woodward JE, Miller NA, Schilkey FD, et al. C6/36 Aedes albopictus cells have a dysfunctional antiviral RNA interference response. PLoS Negl Trop Dis. 2010;4:e856.
14. R Development Core Team. R: A language and environment for statistical computing. Vienna: R Foundation for Statistical Computing; 2016. https:// www.R-project.org/

15. Lê $S$, Josse J, Husson F. FactoMineR: an R package for multivariate analysis. I Stat Softw. 2008;25:1-18.

16. Chong J, Soufan O, Li C, Caraus I, Li S, Bourque G, et al. MetaboAnalyst 4.0: towards more transparent and integrative metabolomics analysis. Nucleic Acids Res. 2018;46:W486-94.

17. Schwammle V, Jensen ON. VSClust: feature-based variance-sensitive clustering of omics data. Bioinformatics. 2018;34:2965-72.

18. Mourao MP, Bastos Mde S, de Figueiredo RP, Gimaque JB, Galusso Edos S, Kramer VM, et al. Mayaro fever in the city of Manaus, Brazil, 2007-2008. Vector Borne Zoonotic Dis. 2012;12:42-6.

19. Mavian C, Rife BD, Dollar JJ, Cella E, Ciccozzi M, Prosperi MCF, et al. Emergence of recombinant Mayaro virus strains from the Amazon basin. Sci Rep. 2017:7:8718.

20. Mota MTO, Ribeiro MR, Vedovello D, Nogueira ML. Mayaro virus: a neglected arbovirus of the Americas. Future Virol. 2015;10:1109-22.

21. Rossmann MG, Rao VB. Viral molecular machines, vol. 726. Boston: Springer; 2011.

22. Hidari KI, Suzuki T. Dengue virus receptor. Trop Med Health. 2011;39(Suppl. 4):S37-43.

23. Wintachai P, Wikan N, Kuadkitkan A, JaimipukT, Ubol S, Pulmanausahakul $\mathrm{R}$, et al. Identification of prohibitin as a chikungunya virus receptor protein. J Med Virol. 2012:84:1757-70.

24. Gething MJ, Sambrook J. Protein folding in the cell. Nature. 1992;355:33.

25. Young RA. Stress proteins and immunology. Annu Rev Immunol. 1990;8:401-20

26. da Costa Carvalho MG, Fournier MV. Effect of heat shock on gene expression of Aedes albopictus cells infected with Mayaro virus. Res Virol. 1991;142:25-31.

27. Lee RC, Chu JJ. Proteomics profiling of chikungunya-infected Aedes albopictus $\mathrm{C6/36}$ cells reveal important mosquito cell factors in virus replication. PLoS Negl Trop Dis. 2015;9:e0003544.

28. Merkling SH, Overheul GJ, van Mierlo JT, Arends D, Gilissen C, van Rij RP. The heat shock response restricts virus infection in Drosophila. Sci Rep. 2015:5:12758.

29. Iwasaki S, Kobayashi M, Yoda M, Sakaguchi Y, Katsuma S, Suzuki T, et al. $\mathrm{Hsc70/Hsp90} \mathrm{chaperone} \mathrm{machinery} \mathrm{mediates} \mathrm{ATP-dependent} \mathrm{RISC} \mathrm{load-}$ ing of small RNA duplexes. Mol Cell. 2010:39:292-9.

30. Sim C, Hong YS, Tsetsarkin KA, Vanlandingham DL, Higgs S, Collins FH. Anopheles gambiae heat shock protein cognate 70B impedes o'nyongnyong virus replication. BMC Genomics. 2007;8:231.

31. Miyazaki JH, Yang SF. The methionine salvage pathway in relation to ethylene and polyamine biosynthesis. Physiol Plant. 1987;69:366-70.

32. Ge C, Wan D, Wang Z, Ding Y, Wang Y, Shang Q, et al. A proteomic analysis of rice seedlings responding to 1,2,4-trichlorobenzene stress. Int J Environ Sci. 2008;20:309-19.

33. Patramool S, Surasombatpattana P, Luplertlop N, Seveno M, Choumet V, Thomas F, et al. Proteomic analysis of an Aedes albopictus cell line infected with dengue serotypes 1 and 3 viruses. Parasit Vectors. 2011:4:138.

34. Fontaine KA, Sanchez EL, Camarda R, Lagunoff M. Dengue virus induces and requires glycolysis for optimal replication. J Virol. 2015;89:2358-66.

35. Jose J, Taylor AB, Kuhn RJ. Spatial and temporal analysis of alphavirus replication and assembly in mammalian and mosquito cells. mBio. 2017;8:e02294-16.

36. Ritter JB, Wahl AS, Freund S, Genzel Y, Reichl U. Metabolic effects of influenza virus infection in cultured animal cells: intra- and extracellular metabolite profiling. BMC Syst Biol. 2010;4:61.

37. Bhattacharya S, Stewart BA, Niemeyer BA, Burgess RW, McCabe BD, Lin $P$, et al. Members of the synaptobrevin/vesicle-associated membrane protein (VAMP) family in Drosophila are functionally interchangeable in vivo for neurotransmitter release and cell viability. Proc Natl Acad Sci USA. 2002:99:13867-72.

38. Enzmann PJ. Induction of an interferon-like substance in persistently infected Aedes albopictus cells. Arch Gesamte Virusforsch. 1973:40:382-9.

39. Vogel JL, Kristie TM. The dynamics of HCF-1 modulation of herpes simplex virus chromatin during initiation of infection. Viruses. 2013;5:1272-91.

40. Freiman RN, Herr W. Viral mimicry: common mode of association with HCF by VP16 and the cellular protein LZIP. Genes Dev. 1997;11:3122-7. 
41. Goic B, Vodovar N, Mondotte JA, Monot C, Frangeul L, Blanc H, et al. RNA-mediated interference and reverse transcription control the persistence of RNA viruses in the insect model Drosophila. Nat Immunol. 2013;14:396-403.

42. Perez-Riverol Y, Alpi E, Wang R, Hermjakob H, Vizcaino JA. Making proteomics data accessible and reusable: current state of proteomics databases and repositories. Proteomics. 2015;15:930-49.

43. Jarnuczak AF, Vizcaino JA. Using the PRIDE database and proteomeXchange for submitting and accessing public proteomics datasets. Curr Protoc Bioinformatics. 2017;59:13-31.
44. Vizcaino JA, Deutsch EW, Wang R, Csordas A, Reisinger F, Rios D, et al. ProteomeXchange provides globally coordinated proteomics data submission and dissemination. Nat Biotechnol. 2014;32:223-6.

\section{Publisher's Note}

Springer Nature remains neutral with regard to jurisdictional claims in published maps and institutional affiliations.
Ready to submit your research? Choose BMC and benefit from:

- fast, convenient online submission

- thorough peer review by experienced researchers in your field

- rapid publication on acceptance

- support for research data, including large and complex data types

- gold Open Access which fosters wider collaboration and increased citations

- maximum visibility for your research: over $100 \mathrm{M}$ website views per year

At BMC, research is always in progress.

Learn more biomedcentral.com/submissions 